\title{
Eher Agentur als Amt
}

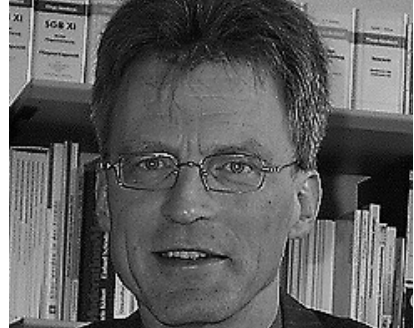

VON THOMAS DANE

Thomas Dane ist Vorstand des Diakonischen Werkes BerlinBrandenburg-schlesische Oberlausitz e. V.

E-Mail dane.t@dwbo.de
Wenn sich Zuständigkeiten im Sozialbereich von der Landes- auf die kommunale Ebene verschieben, muss dies auch Auswirkungen auf die Organisationsstruktur von regionalen Wohlfahrtsverbänden haben. Anstelle der strikten Gliederung nach fachlichen Zuständigkeiten sollten kleine Teams treten, die auftragsorientiert weitere Kompetenzen hinzuziehen können.

In den letzten Jahren hat es in der Sozialpolitik zwei gegenläufige Entwicklungen gegeben. Während im Bereich der Krankenhäuser und Pflegeeinrichtungen Regelungskompetenzen von der Landes- an die Bundesebene abgegeben worden sind, werden insbesondere Zuständigkeiten der Sozialhilfe von der Landes- auf die kommunale Ebene übertragen. Die Auswirkungen auf Wohlfahrtsverbände und Leistungserbringer lassen sich am Beispiel der Kommunalisierung der Zuständigkeit für die Eingliederungshilfe im Land Brandenburg beschreiben.

Am 31. Dezember 2006 trat $\mathbb{1 0 0}$ Abs. 1 des alten Bundessozialhilfegesetzes (BSHG) außer Kraft. Dadurch ergab sich ab Januar 2007 eine neue rechtliche Grundlage für die Zuständigkeit des überörtlichen Trägers der Sozialhilfe. Nach $\mathbb{9} 97$ Abs. 1 SGB XII ist für die Sozialhilfe sachlich der örtliche Träger zuständig, soweit nicht der überörtliche Träger sachlich zuständig ist. Nach $\mathbb{9} 97$ Abs. 2 SGB XII wird die sachliche Zuständigkeit des überörtlichen Trägers der Sozialhilfe nach Landesrecht bestimmt.

In Brandenburg ist den Kommunen als örtlichen Trägern der Sozialhilfe mit dem am 6. Dezember 2006 beschlossenen Ausführungsgesetz zum SGB XII die Zuständigkeit auch für die stationäre Eingliederungshilfe und Hilfe zur Pflege übertragen worden. Zugleich wurden durch Änderung des Brandenburgischen Finanzausgleichsgesetzes den Kommunen als örtlichen Trägern der Sozialhilfe zur Erfüllung ihrer Aufgaben ab dem Jahr 2007 entsprechende Haushaltsmittel im Rahmen des allgemeinen Finanzausgleiches zur Verfügung gestellt.

Mit Urteil vom 15. Dezember 2008 hat das Brandenburgische Verfassungsgericht diese Regelung des Brandenburgischen Finanzausgleichsgesetzes für verfassungswidrig erklärt. In den Urteilsbegründungen hat das Landesverfassungsgericht ausgeführt, dass das Recht auf kommunale Selbstverwaltung nach Art. 97 Abs. 3 der Landesverfassung in seiner Ausprägung durch das Konnexitätsprinzip das Land zum Ausgleich der Mehrbelastungen verpflichte, die den Gemeinden dadurch entstehen, dass ihnen das Land neue Aufgaben überträgt. Das Verfassungsgericht hat dem Land aufgegeben, spätestens für das Haushaltsjahr 2010 für den gesamten Aufgabenbereich eine gesonderte, Finanzkraft unabhängige Kostenerstattungsregelung zu treffen.

Die Wohlfahrtsverbände im Land Brandenburg hatten sich im Jahr 2006 gegen die Kommunalisierung der Sozialhilfe im Land Brandenburg ausgesprochen. Dabei befürchteten sie zum einen die Zweckentfremdung von Landeszuweisungen, die eigentlich zur Deckung von Sozialhilfeausgaben gedacht waren. Zum anderen befürchteten sie stark voneinander abweichende Planungs- und Finanzierungsgrundlagen in den 14 Landkreisen und vier kreisfreien Städten und damit eine Differenzierung der Lebensverhältnisse in Brandenburg.

Es zeigte sich jedoch schnell, dass es unter den politischen Entscheidungsträgern in Brandenburg ein hohes Einvernehmen im Hinblick auf die Kommunalisierung der Eingliederungshilfe gab. Zudem gab es aus Sicht der Wohlfahrtsverbände keinen vernünftigen Grund, die Zusammenführung der bisher beim örtlichen Sozialhilfeträger liegenden Fachverantwortung mit der beim überörtlichen Sozialhilfeträger liegenden Finanzverantwortung für stationäre Hilfen im Rahmen der Eingliederungshilfe und der Hilfe zur Pflege zu blockieren. Für ambulante Hilfen lag die Fach- und Finanzverantwortung bereits vor 2007 beim örtlichen Sozialhilfeträger.

Die Zuständigkeitsverteilung in der Sozialhilfe vor 2007 hatte zur Folge, dass im Bundesvergleich in Brandenburg die ambulanten Leistungen sehr zurückhaltend gewährt wurden. Denn das knappe Angebot an ambulanten Leistungen führte dazu, dass verstärkt stationäre Betreuungsformen in Anspruch genommen wurden. Da aber 
stationäre Betreuungsformen häufig teurer als ambulante Betreuungsformen sind, führte dies zu vergleichsweise hohen Sozialhilfeausgaben.

Insofern war die Zusammenführung der Fach- und Finanzverantwortung für alle Betreuungsformen auf kommunaler Ebene konsequent. Zunächst war daran gedacht, die Zuständigkeit für die Vertragsgestaltung mit den Leistungserbringern und die Vereinbarung von Leistungsentgelten beim überörtlichen Träger der Sozialhilfe zu belassen. Dieser hätte dann im kommunalen Auftrag die Leistungsentgelte mit den Leistungserbringern verhandelt.

Aus Sicht der Wohlfahrtsverbände barg diese Aufgabenverteilung das Risiko dreiseitiger Verhandlungen, die sich bereits vor 2007 aufgrund der oft konfliktbehafteten Zusammenarbeit zwischen den örtlichen und dem überörtlichen Träger der Sozialhilfe als sehr schwierig herausgestellt hatten. Eine konkurrierende Steuerung der Sozialhilfeausgaben durch Land und Kommunen erweist sich unter der Bedingung knapper öffentlicher Mittel als nachteilig für die Leistungserbringer.

Es entsprach daher dem Wunsch der Wohlfahrtsverbände, dass sich Ende 2006 bis auf einen Landkreis alle Landkreise und kreisfreien Städte in Brandenburg entschlossen, eine gemeinsame Serviceeinheit für die Vertragsgestaltung mit den Leistungserbringern und die Vereinbarung von Leistungsentgelten zu errichten.

Im Zeitraum von 2007 bis 2009 haben die Wohlfahrtsverbände mit den Landkreisen und kreisfreien Städten gut zusammengearbeitet. Dies lag zum einen an der gemeinsamen Serviceeinheit der Landkreise und kreisfreien Städte, zum anderen an einem sehr verlässlichen Abstimmungsverfahren der Landkreise und kreisfreien Städte in einer gemeinsamen Steuerungsgruppe. Diese Steuerungsgruppe sicherte die Beteiligung aller Landkreise und Städte am Abstimmungsverfahren, aber auch klare Ansprechstrukturen. Zudem war die gemeinsame Verhandlungskommission, der Brandenburger Ausschuss, vergleichsweise klein, was eine vertrauensvolle $\mathrm{Zu}$ sammenarbeit erleichtert.

Nicht übersehen werden darf, dass der 2007 bis 2009 vom Land zur Verfügung gestellte Finanzrahmen auskömmlich war, was die Zusammenarbeit ebenfalls erleichterte.

Auch die Zusammenarbeit zwischen den Leistungserbringern und den einzelnen örtlichen Sozialhilfeträgern war bis auf wenige
Ausnahmen gut. Entgegen ursprünglicher Befürchtungen versuchte nur ein Landkreis untergebrachte Personen, die zuvor ihren Wohnsitz in einem anderen Landkreis hatten, wieder in den Herkunftslandkreis zurückzuführen. Die Wohlfahrtsverbände haben sich nur dort unmittelbar auf örtlicher Ebene für die ihnen angeschlossenen Leistungserbringer engagiert, wo es Probleme gab: im Berichtszeitraum in einem von 14 Landkreisen und einer von vier kreisfreien Städten.

\section{Risiken und Nebenwirkungen}

Mit dem Beschluss des Brandenburgischen Verfassungsgerichtes vom 15. Dezember 2008 zur Rechtswidrigkeit der jetzigen Regelung wegen Verstoßes gegen das Konnexitätsprinzip bedurften das Ausführungsgesetz zum SGB XII und das Brandenburgische Finanzausgleichsgesetz zum 1. Januar 2010 der Neugestaltung. Anders als 2006 befürworten die Wohlfahrtsverbände heute die Kommunalisierung der $\mathrm{Zu}$ ständigkeiten in der Sozialhilfe.

Diese Position ist nicht frei von Risiken. Kommunen und Wohlfahrtsverbände haben ein gemeinsames Interesse an der Bereitstellung eines auskömmlichen Finanz-

\section{Organisationsplan DWBO (gültig ab 01.07.2008)}

\section{- Presse-/Öffentlichkeitsarbeit, Ökumene/Brot für die Welt Christiane Lehmacher-Dubberke \\ - Int. Organisation \\ - Mitarbeitervertretung \\ - Projekt Känguru}

AB 3: Kindertageseinrichtungen,

Geschäftsstelle des Verbandes Evang. Tageseinrichtungen für Kinder (VETK) Jürgen Schwochow

AB 4: Gesundheit, Geschäftsstelle des Verbandes Evang. Krankenhäuser und stationärer Pflegeeinrichtungen (VEKP) Detlef Albrecht

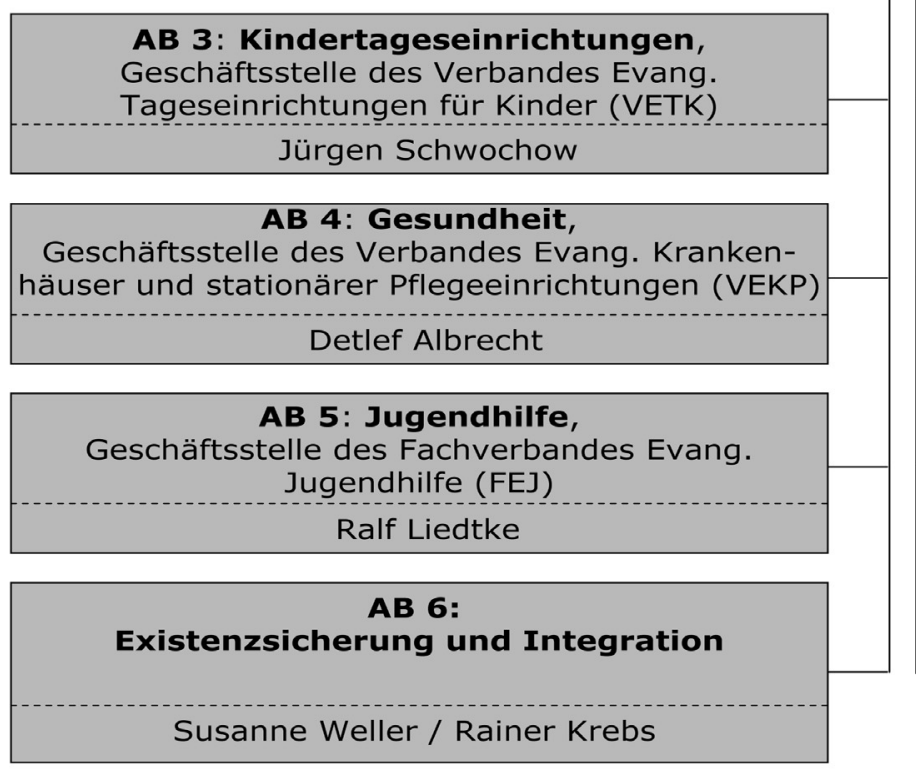

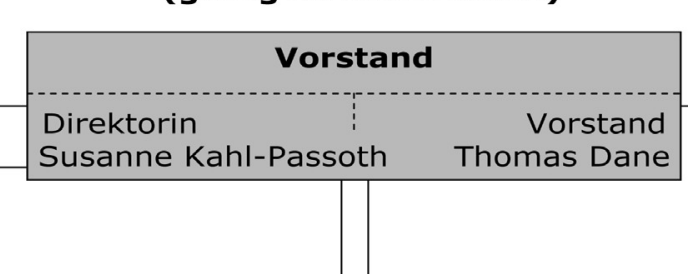

- Arbeitsrecht: AGMV, dgv, AK-DWBO, Schieds-/Schlichtungsst. - Personal

- Fortbildung

- EDV

- Vermögensverwaltung

- Projekt Patenmodell

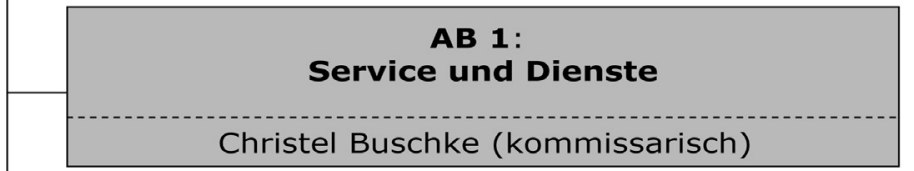

Die interne Organisation eines Landeswohlfahrtsverbandes wie dem Diakonischen Werk Berlin-Brandenburg-schlesische Oberlausitz e. V. (DWBO) muss heute so aufgebaut sein, dass personelle Ressourcen flexibel und angepasst an den akuten Bedarf eingesetzt werden können. Neue Herausforderungen wie die Kommunalisierung von sozialen Aufgaben erfordern andere Organisationskonzepte etwa in Anlehnung an die Arbeitsweise von Unternehmensberatungen oder Rechtsanwaltskanzleien. Es braucht kleine Teams mit Kernkompetenzen, die bei Bedarf andere im Verband vorhandene Kompetenzen hinzuziehen können, und auftrags- oder mandantenorientiert arbeiten. 
rahmens für die Leistungen der Eingliederungshilfe und der Hilfe zur Pflege. Sollte das Land eine erhebliche Kürzung dieses Finanzrahmens durchsetzen, würde dies das Verhältnis von Kommunen und Wohlfahrtsverbänden erheblich belasten und die Zahl der Konflikte zwischen örtlichen Sozialhilfeträgern und Leistungserbringern würde zunehmen.

Die aktuelle Wirtschaftskrise und deren Auswirkungen auf das Steueraufkommen von Land und Kommunen werden in diesem Sinne zu Belastungen führen. Es wird sich deshalb in diesem Jahr und den Folgejahren zeigen, wie belastbar das Verhältnis
Zudem halten die Wohlfahrtsverbände auf Landesebene Fachleute für die einzelnen Arbeitsfelder vor. Ein Spezialist für Suchthilfe kann sein Spezialwissen aber nur sehr beschränkt einsetzen, wenn sich seine Tätigkeit im Wesentlichen auf eine Region beschränkt. Reagieren die Wohlfahrtsverbände auf die Kommunalisierung von Zuständigkeiten mit einer Personalverschiebung auf die regionale Ebene, riskieren sie auf Landesebene einen Kompetenzverlust, ohne dass die Kompetenzen auf regionaler Ebene angemessen genutzt werden können. Auch wäre diese Strategie nur mittels einer gravierenden Personalverstärkung umsetzbar.

\section{"Auf kommunaler Ebene haben persönliche Kontakte hohe Bedeutung"}

von Land und Kommunen zwischenzeitlich geworden ist. Bisher ist es den Wohlfahrtsverbänden gelungen, sich erfolgreich mit der Kommunalisierung der Eingliederungshilfe zu arrangieren.

Die Liga der Spitzenverbände der Freien Wohlfahrtspflege verfügt in Brandenburg über eine regionale Struktur: die sogenannten Kleinen Ligen, das sind die Zusammenschlüsse der Wohlfahrtsverbände in den Landkreisen und kreisfreien Städten. Während jedoch auf Landesebene die Interessenvertretung der Leistungserbringer über jeweils mehrere hauptamtliche Beschäftigte der Landesverbände der Freien Wohlfahrtspflege betrieben wird, betreiben die Vertreter in den Kleinen Ligen die Interessenvertretung überwiegend als $\mathrm{Ne}$ bentätigkeit. Auch gibt es pro Verband und Region selten mehr als eine Person, die in die Interessenvertretung eingebunden ist. Während die Wohlfahrtsverbände auf Landesebene über eine größere Zahl von Fachleuten verfügen, verfügen sie auf regionaler Ebene eher über wenige » Laien «.

Es besteht nun die Möglichkeit, die regionale Interessenvertretung personell besser auszustatten, also Fachleute von der Landesebene auf die regionale Ebene herunterzuholen. Die Wohlfahrtsverbände nehmen die Interessenvertretung jedoch nicht arbeitsteilig wahr, sondern einzelverbandlich. Kein einzelner Wohlfahrtsverband ist aber in der Lage, in jeder Region einen hauptamtlichen Interessenvertreter vorzuhalten. Insofern führt eine Personalverschiebung von der Landes- auf die regionale Ebene zu Verwerfungen in der Leistungsfähigkeit der Verbände auf regionaler Ebene.
Versuchen Wohlfahrtsverbände umgekehrt von der Landesebene aus die regionale Interessenvertretung zu betreiben, dann wird es dem Mitarbeiter eines Landesverbandes schwerfallen, sich in die örtlichen Kommunikationsnetze einzufädeln. Er bleibt vor Ort ein Außenstehender, der schon deshalb als Interessenvertreter nicht hilfreich sein kann. Auf kommunaler Ebene sind Kommunikations- und Verhandlungsstrukturen weniger formell als auf Landesebene und die Bedeutung persönlicher Kontakte und Beziehungen ist höher. Dies erfordert eine Präsenz vor Ort.

\section{Leistungserbringer an die Front!}

In Brandenburg haben die Wohlfahrtsverbände auf die Kommunalisierung der Eingliederungshilfe anders reagiert. Sie haben die Leistungserbringer in der Region aufgefordert, ihre Interessen gegenüber dem örtlichen Sozialhilfeträger selbst wahrzunehmen. Die Kleinen Ligen wurden eher angehalten, die Zusammenarbeit der Leistungserbringer mit dem örtlichen Sozialhilfeträger nicht zu stören. Dem liegt die Einschätzung zugrunde, dass Leistungserbringer ihre Interessen vor Ort professioneller selbst vertreten können, als sich durch nebenamtliche Interessenvertreter vertreten zu lassen. Die Landesverbände haben den Leistungserbringern aber Unterstützung angeboten:

- die Moderation der Meinungsbildung unter den Leistungserbringern, wenn zwischen diesen Interessenkonflikte bestehen

- die Übernahme der Verhandlungsführung in der Region, wenn der örtliche
Sozialhilfeträger einen aggressiven Konfliktkurs fährt

- die Entwicklung von Vertragsentwürfen für Verhandlungen vor Ort

- die Sicherstellung der Information über Verhandlungsergebnisse in anderen Regionen

- die umfassende fachliche, betriebswirtschaftliche, rechtliche, strategische und taktische Beratung der Leistungserbringer mit Blick auf die Verhandlungen mit dem örtlichen Sozialhilfeträger

- die Sicherstellung des Erfahrungsaustausches unter den Leistungserbringern.

Insbesondere ist es den Wohlfahrtsverbänden in Brandenburg und den anderen Beteiligten aber gelungen, in Reaktion auf die Kommunalisierung der Eingliederungshilfe mit dem Brandenburger Ausschuss eine neue Verhandlungsplattform mit enger Bindung an die kommunale Ebene zu schaffen. Diese Plattform wurde zudem genutzt, um informelle Kontakte in die Kommunen hinein zu entwickeln und zu pflegen. $\mathrm{Zu}$ dem wurden so gezielt die Meinungsführer in den Kommunen erreicht.

\section{Organisationsentwicklung auf Landesebene notwendig}

Diese Arbeitsweise ist Landesverbänden der Freien Wohlfahrtspflege aber nur möglich, wenn sie ihre personellen Ressourcen flexibel und angepasst an den akuten Bedarf einsetzen können. Mit diesen Erfordernissen ist eine starre Organisationsstruktur von Verbänden nicht vereinbart, die Organisationseinheiten strikt nach fachlichen Zuständigkeiten bildet und abgrenzt. Dies ist aber die traditionelle Organisationsstruktur von Wohlfahrtsverbänden, die insofern die Organisation der öffentlichen Verwaltung spiegelt.

Neue Herausforderungen wie die Kommunalisierung von sozialen Aufgaben erfordern andere Organisationskonzepte etwa in Anlehnung an die Arbeitsweise von Unternehmensberatungen oder Rechtsanwaltskanzleien. Es braucht kleine Teams mit Kernkompetenzen, die bei Bedarf andere im Verband vorhandene Kompetenzen hinzuziehen können, und auftrags- oder mandantenorientiert arbeiten.

Im Land Brandenburg sind auch andere soziale Aufgaben auf die kommunale Ebene übertragen worden. Anders als im Bereich der Eingliederungshilfe haben die Wohlfahrtsverbände in den Bereichen der Arbeitsförderung und der Erziehungshilfe bisher kein Konzept gefunden, erfolgreich auf die Kommunalisierung zu reagieren. Dagegen ist die Zuständigkeit im Bereich der Kindertagesstätten geteilt: Hier scheint es zu gelingen, für die Zukunft eine funktionsfähige Interessenvertretung zu organisieren. 